\section{Covid-19: Suicide rates were static in rich countries in first months of pandemic, study finds}

\author{
Jacqui Wise
}

Numbers of suicides in high and upper middle income countries remained largely unchanged or were lower than expected levels in the early months of the covid-19 pandemic, shows a study in the Lancet Psychiatry. ${ }^{1}$

The authors said that many countries put in place additional mental health supports and financial safety nets that may have helped to ameliorate early adverse effects of the pandemic. However, they emphasised that governments needed to remain vigilant and be poised to respond if the situation changes as the longer term mental health and economic effects of the pandemic unfold.

The study looked at numbers of suicides in 21 high income and upper middle income countries and regions between 1 April and 31 July 2020. Researchers used real time data on suicide numbers obtained from official government sources and compared them with trends in suicides in the previous 1-4 years. The study included national data for 10 countries and data from 25 specific areas in 11 countries.

It found no evidence of an increase in suicide numbers in any of the countries included. In 12 countries or areas there was evidence of lower than expected suicide numbers (box 1).

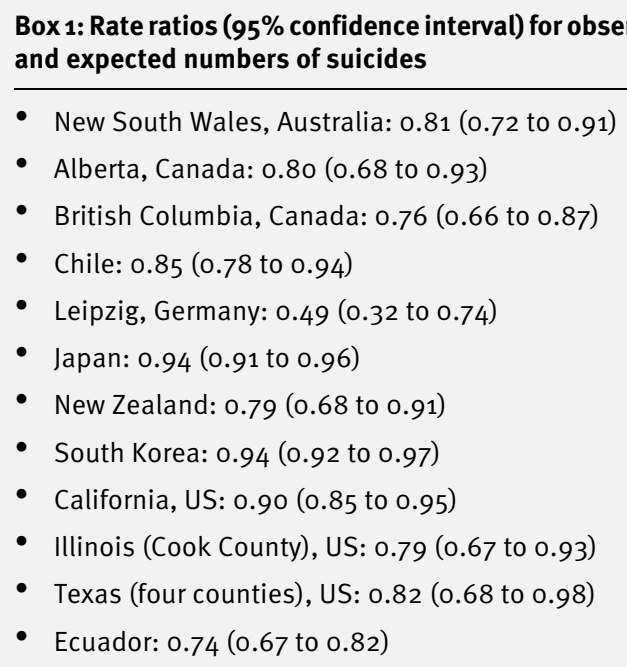

The study had around 70 authors from 30 countries who are members of the International COVID-19 Suicide Prevention Research Collaboration, which was established to monitor the effect of the pandemic on suicide and suicidal behaviour around the world and to advise on ways to mitigate any risks.

The authors suggested several reasons for suicide rates not increasing during the pandemic. Communities may have actively tried to support individuals at risk, people may have connected with others in new ways, and some relationships may have been strengthened by households spending more time with each other. For some people, everyday stresses may have been reduced during periods spent at home, and a collective feeling of "we're all in this together" may have benefited some.

The lead author, Jane Pirkis, director of the Centre for Mental Health at the University of Melbourne, said, "We need to continue to monitor the data and be alert to any increases in suicide, particularly as the pandemic's full economic consequences emerge.

"Increasing mental health services and suicide prevention programmes, and providing financial safety nets, may help to prevent the possible longer term detrimental effects of the pandemic on suicide."

She added, "We know that many people have had their lives changed dramatically by the pandemic, and the journey for some of them is ongoing. We need to recognise that suicide is not the only indicator of the negative mental health effects of the pandemic-levels of community distress are high, and we need to ensure that people are supported."

The researchers warned of some signals that the pandemic might be adversely affecting suicide rates in low income and lower middle income countries, although data were available for only a small minority of these countries and tended to be of poor quality.

Writing in a linked commentary, Stella Botchway and Seena Fazel, from the University of Oxford, said, "Despite this initial snapshot, governments and services need to remain vigilant for a possible delayed increase in suicides as a result of the pandemic. Suicide can be a lagging indicator of psychosocial difficulties, influenced by medium-term and longer-term disruptions to civic life and the economy. Other work has shown that suicides can increase following economic recession, and such increases can be sustained for several years.”

The high income countries included in the study were Australia, Austria, Canada, Chile, Croatia, England, Estonia, Germany, Italy, Japan, Netherlands, New Zealand, Poland, South Korea, Spain, and the US. The upper-middle income countries were Brazil, Ecuador, Mexico, Peru, and Russia.

Pirkis J, John A, Shin S, etal. Suicide trends in the early months of the covid-19 pandemic: an interrupted time-series analysis of preliminary data from 21 countries. Lancet Psychiatry 2021. doi: 10.1016/S2215-0366(21)00091-2

This article is made freely available for use in accordance with BMJ's website terms and conditions for the duration of the covid-19 pandemic or until otherwise determined by BMJ. You may use, download and print the article for any lawful, non-commercial purpose (including text and data mining) provided that all copyright notices and trade marks are retained. 